\title{
A common fixed point theorem for nonself mappings
}

\author{
Vasile Berinde
}




\title{
A COMMON FIXED POINT THEOREM FOR NONSELF MAPPINGS
}

\author{
VASILE BERINDE
}

[Received: October 23, 2003]

\begin{abstract}
A general common fixed point theorem for nonself discontinuous mappings is given. It contains, as particular cases, many classical and recent results in fixed point theory.

Mathematics Subject Classification: 47H10

Keywords: Banach space, generalized quasi contraction, common fixed point, nonself mappings
\end{abstract}

\section{InTRODUCTION}

Banach's fixed point theorem is one of the most useful results in fixed point theory. In a metric space setting it can be briefly stated as follows.

Theorem B. Let $(X, d)$ be a complete metric space and $T: X \rightarrow X$ a strict contraction, i. e., a map satisfying

$$
d(T x, T y) \leq \alpha d(x, y), \quad \forall x, y \in X,
$$

where $0<\alpha<1$ is a constant. Then $T$ has a unique fixed point in $X$.

Theorem B, together with its local variants, has many applications in solving nonlinear functional equations, but has one drawback the contraction condition (1.1) forces $T$ to be continuous on the entire $X$.

In 1968, Kannan [6] obtained a fixed point theorem for mappings $T$ that need not be continuous.

Theorem K. Let $(X, d)$ be a complete metric space and $T: X \rightarrow X$ a mapping for which there exists $a \in\left(0, \frac{1}{2}\right)$ such that

$$
d(T x, T y) \leq a[d(x, T x)+d(y, T y)], \text { for all } x, y \in X .
$$

Then $T$ has a unique fixed point in $X$.

Example 1. Let $X$ be the set of reals with the usual norm and $T: X \rightarrow X$ given by $T x=0$ if $x \in(-\infty, 2]$, and $T x=-\frac{1}{2}$ if $x \in(2, \infty)$. Then $T$ satisfies (1.2) with $a=\frac{1}{5}$ and $T$ is not continuous. 
Following Kannan's theorem, a great many papers were devoted to obtaining fixed point theorems for various contractive conditions that do not require the continuity of $T$, see, for example, Rus [11].

One of the most general contractive conditions obtained in this way, for which the Picard iteration still converges to the unique fixed point, was given by Ćirić [4].

Theorem C1. Let $(X, d)$ be a complete metric space and $T: X \rightarrow X$ a mapping such that

$$
d(T x, T y) \leq h \cdot \max \{d(x, y), d(x, T x), d(y, T y), d(x, T y), d(y, T x)\}
$$

for all $x, y \in X$ and for some constant $0<h<1$.

Then $T$ has a unique fixed point in $X$.

Remarks. $\mathbf{1}^{\circ}$ As shown by Rhoades [10, Theorem 2], a contractive mapping satisfying (1.3) is still continuous at the fixed point.

$\mathbf{2}^{\circ}$ The fixed point theorems for contractive definitions of the form (1.1)-(1.3) were unified by many authors, see for example Berinde [1], Rus [11]. For a recent comparison of various contractive type conditions we refer to Mészáros [7].

$\mathbf{3}^{\circ}$ The set $0_{T}(x)=\left\{x, T x, T^{2} x, \ldots\right\}$ is called the orbit of $T$ relative to $x$. It is shown in [12] that condition (1.3), in fact, ensures that the orbits of $T$ are bounded.

For any $T: X \rightarrow X$ and $x, y \in X$, where $X$ is a metric space, let us put

$$
\begin{aligned}
B(x, y) & =d(x, y) ; \\
K(x, y) & =\frac{1}{2}[d(x, T x)+d(y, T y)] ; \\
C(x, y) & =\max \{d(x, y), d(x, T x), d(y, T y), d(x, T y), d(y, T x)\} .
\end{aligned}
$$

The following theorem formally unifies Banach's, Kannan's and Ćirić's fixed point theorems.

Theorem G. Let $(X, d)$ be a complete metric space and $T: X \rightarrow X$ a mapping satisfying

$$
d(T x, T y) \leq \lambda E(x, y) \text { for all } x, y \in X
$$

where $\lambda$ is a constant, $0<\lambda<1$, and $E(x, y)$ is any of the expressions $B(x, y), K(x, y)$ and $C(x, y)$.

Then $T$ has a unique fixed point.

Remarks. $\mathbf{1}^{\circ}$ Theorem $\mathrm{G}$ above can be extended by considering a function $\varphi$ : $\mathbb{R}_{+} \rightarrow \mathbb{R}_{+}$( $\mathbb{R}_{+}$denotes the set of nonnegative numbers) which preserves some essential properties of the function

$$
\varphi(t)=\lambda t, \quad t \in \mathbb{R}_{+} \quad(0<\lambda<1)
$$

appearing in (1.4) and by replacing condition (1.4) by a more general one:

$$
d(T x, T y) \leq \varphi(E(x, y)) \text { for all } x, y \in X .
$$


$2^{\circ}$ One of the first results of this kind was obtained by Browder [3]. The function $\varphi$ involved in such fixed point theorems is usually called comparison function and is supposed to satisfy at least the following two conditions:

$\left(\mathrm{i}_{\varphi}\right) \varphi$ is nondecreasing, i. e., $t_{1}<t_{2} \Rightarrow \varphi\left(t_{1}\right) \leq \varphi\left(t_{2}\right)$;

(ii $\left.\varphi_{\varphi}\right)$ The sequence $\left\{\varphi^{n}(t)\right\}$ converges to zero for every $t \in \mathbb{R}_{+}$, where $\varphi^{n}$ stands for the $n$th iterate of $\varphi$.

Example 2. It is easy to check that a comparison function $\varphi$ needs to be neither linear nor continuous, by considering $\varphi_{1}(t)=\frac{t}{1+t}, t \in \mathbb{R}_{+}$and $\varphi_{2}(t)=\frac{t}{2}$ if $0 \leq t<1$ and $\varphi_{2}(t)=t-\frac{1}{3}$ if $t \geq 1$.

To prove our main result we shall need the following Lemma.

Lemma 1. If $\varphi$ satisfies $\left(\mathrm{i}_{\varphi}\right)$ and $\left(\mathrm{ii}_{\varphi}\right)$ and is such that

$$
t \leq \varphi(t) \quad \text { for a certain } t \in \mathbb{R}_{+},
$$

then $t=0$.

Proof. Suppose the contrary, i. e., there exists $t>0$ such that (1.7) is satisfied. Then, by induction, in view of $\left(\mathrm{ii}_{\varphi}\right)$, we get

$$
t \leq \varphi^{n}(t), \quad n \geq 1 .
$$

By virtue of ( $\left.\mathrm{ii}_{\varphi}\right)$, this implies that $t \leq \varphi^{n}(t) \rightarrow 0$ as $n \rightarrow \infty$, a contradiction.

\section{Contractions that aRE NOT SELF-MAPPINGS}

All fixed point theorems stated in the previous section deal with a self-mapping of a metric space. However, in many applications of fixed point theory, either a mapping of a closed subset $K$ of $X$ is not a self-mapping of $K$ or it is very difficult to verify the invariance condition $T(K) \subset K$.

It was thus an open problem for more than 20 years to extend Theorem $\mathrm{C} 1$ from self-mappings $T: K \rightarrow K$ satisfying (1.3) to the corresponding nonself-mappings $T: K \rightarrow X$, where $K \neq X$. Recently, Ćirić [5] solved this problem by considering an additional boundary condition, also known as Rothe's boundary condition, which, however, restricts his results to a Banach space setting.

Theorem C2. Let $E$ be a Banach space, $K$ a nonempty closed subset of $E$, and $\partial K$ the boundary of $K$. Let $T: K \rightarrow E$ be a nonself-mapping satisfying (1.3) for all $x, y \in K$. If

$$
T(\partial K) \subset K,
$$

then $T$ has a unique fixed point in $K$.

Very recently, Theorem $\mathrm{C} 2$ was extended by Rakočević [9] to a common fixed point theorem. Radovanovic [8] also considered a similar but more particular contractive condition. 
The main aim of this paper is to unify the results of Ćirić and Rakočević, as well as many other related results, in the framework of a very general common fixed point theorem.

\section{Main Result}

Let $E$ be a normed linear space. For $x, y \in E$ we shall denote by

$$
\operatorname{seg}[x, y]=\{z \in E: z=(1-t) x+t y, \quad 0 \leq t \leq 1\}
$$

the segment of extremities $x$ and $y$. The proof of the next lemma is straightforward, see Rakočević [9].

Lemma 2. If $u \in E$ and $z \in \operatorname{seg}[x, y]$, then

$$
\|u-z\| \leq \max \{\|u-x\|,\|u-y\|\} .
$$

Now we can state the main result of this paper.

Theorem 1. Let $E$ be a Banach space, $K$ a nonempty closed subset of $E$ and $\partial K$ the boundary of $K$. Let $S: K \rightarrow E$ and $T: E \rightarrow E, T: K \rightarrow K$. Suppose that $\partial K \neq \varnothing, T$ is continuous, and that $S$ and $T$ satisfy the following conditions:

$1^{\circ}$ There exists a continuous comparison function $\varphi$ such that, for every $x, y \in K$,

$$
d(S x, S y) \leq \varphi(M(x, y)),
$$

where

$$
M(x, y)=\max \{d(T x, T y), d(T x, S x), d(T y, S y), d(T x, S y), d(T y, S x)\}
$$

$\mathbf{2}^{\circ} T$ and $S$ are weakly commutative, i. e.,

$$
d(T S x, S T x) \leq d(T x, S x), \quad \text { for every } \quad x \in K,
$$

and, moreover,

$$
\begin{gathered}
S(K) \cap K \subset T(K), \\
S(\partial K) \subset K
\end{gathered}
$$

and

$$
T(\partial K) \supset \partial K
$$

Then $T$ and $S$ have a unique common fixed point, provided that $T$ and $S$ have bounded orbits.

Proof. Let $x_{0} \in \partial K$. Then $S x_{0} \in K$ by (3.5) and by (3.4) it results that there exists $x_{1} \in K$ such that $T x_{1}=S x_{0}$.

Consider $S x_{1}$. If $S x_{1} \in K$, (3.4) again implies there exists $x_{2} \in K$ such that $T x_{2}=S x_{1}$. If $S x_{1} \notin K$, then by (3.6) there exists $x_{2} \in \partial K$ such that $T x_{2} \in \partial K \cap$ seg $\left[T x_{1}, S x_{1}\right]$. Hence, by induction, we construct a sequence $\left\{x_{n}\right\}$ of points in $K$ as follows. 
If $S x_{n} \in K$, then $T x_{n+1}=S x_{n}$ for some $x_{n+1} \in K$, by (3.4). If $S x_{n} \notin K$, then, by (3.6), we can pick $x_{n+1} \in \partial K$ such that

$$
T x_{n+1} \in \partial K \cap \operatorname{seg}\left[T x_{n}, S x_{n}\right] .
$$

We shall prove that both $\left\{T x_{n}\right\}$ and $\left\{S x_{n}\right\}$ are Cauchy sequences.

Let us first prove that

$$
T x_{n+1} \neq S x_{n} \Rightarrow T x_{n}=S x_{n-1} .
$$

Suppose the contrary, that is, $T x_{n} \neq S x_{n-1}$. Then $x_{n} \in \partial K$ and (3.5) implies $S x_{n} \in K$, i. e. $T x_{n+1}=S x_{n}$, a contradiction. This proves (3.7).

By setting

$$
\begin{aligned}
B(n, k) & =\left\{T x_{j}, S x_{j}: n \leq j \leq n+k\right\}, \\
b(n, k) & =\operatorname{diam}(B(n, k)), \\
B(n) & =\left\{T x_{j}, S x_{j}: n \leq j\right\}, \\
b(n) & =\operatorname{diam}(B(n)),
\end{aligned}
$$

we obtain that $b(n, k) \uparrow b(n)$ as $k \rightarrow \infty$ and $\{b(n)\}$ is a decreasing sequence with positive terms, hence $b=\lim _{n \rightarrow \infty} b(n)$ exists.

In order to prove that $\left\{T x_{n}\right\}$ and $\left\{S x_{n}\right\}$ are Cauchy sequences we must show that $b=0$. We claim that

$$
b(n, k) \leq \varphi(b(n-2, k+2)), n \geq 2, k \geq 0,
$$

and consider the following three cases.

Case 1. $b(n, k)=d\left(T x_{i}, S x_{j}\right)$ with $n \leq i, j \leq n+k$.

If $T x_{i}=S x_{i-1}$, then, by (3.1), we get

$$
b(n, k)=d\left(S x_{i-1}, S x_{j}\right) \leq \varphi\left(M\left(x_{i-1}, x_{j}\right)\right) \leq \varphi(b(n-2, k+2))
$$

because $\varphi$ is monotonically increasing.

If $T x_{i} \neq S x_{i-1}$, then $T x_{i-1}=S x_{i-2}$ and

$$
T x_{i} \in \operatorname{seg}\left[T x_{i-1}, S x_{i-1}\right]=\operatorname{seg}\left[S x_{i-2}, S x_{i-1}\right] \text {. }
$$

Thus,

$$
\begin{aligned}
b(n, k) & =d\left(T x_{i}, S x_{j}\right) \leq \max \left\{d\left(S x_{i-2}, S x_{j}\right), d\left(S x_{i-1}, S x_{j}\right)\right\} \\
& \leq \max \left\{\varphi\left(M\left(x_{i-2}, x_{j}\right)\right), \varphi\left(M\left(x_{i-1}, x_{j}\right)\right)\right\} \\
& =\varphi\left(\max \left\{M\left(x_{i-2}, x_{j}\right), M\left(x_{i-1}, x_{j}\right)\right\}\right) \leq \varphi(b(n-2, k+2)) .
\end{aligned}
$$

Case 2. $b(n, k)=d\left(T x_{i}, T x_{j}\right)$ with $n \leq i, j \leq n+k$.

If $T x_{j}=S x_{j-1}$, then Case 2 reduces to Case 1. If $T x_{j} \neq S x_{j-1}$, then as in Case 1 we have $T x_{j-1}=S x_{j-2}$ and

$$
T x_{j} \in \partial K \cap \operatorname{seg}\left[S x_{j-2}, S x_{j-1}\right] .
$$


Hence,

$$
b(n, k)=d\left(T x_{i}, T x_{j}\right) \leq \max \left\{d\left(T x_{i}, S x_{j-2}\right), d\left(T x_{i}, S x_{j-1}\right)\right\}
$$

and so Case 2 also reduces to Case 1.

Case 3. $b(n, k)=d\left(S x_{i}, S x_{j}\right)$, with $n \leq i, j \leq n+k$.

Then

$$
b(n, k)=d\left(S x_{i}, S x_{j}\right) \leq \varphi\left(M\left(x_{i}, x_{j}\right)\right) \leq \varphi(b(n, k)),
$$

which by Lemma 1 implies $b(n, k)=0$. Hence, due to $S x_{i}=T x_{i}, b(n, k)=$ $d\left(S x_{j}, T x_{i}\right)$, which means Case 1. Therefore, (3.8) is proved.

Now, having in view the continuity of $\varphi$, we let $k \rightarrow \infty$ in (3.8), and obtain

$$
b(n) \leq \varphi(b(n-2)) .
$$

Letting $n \rightarrow \infty$ in the previous inequality we obtain $b \leq \varphi(b)$ which, by Lemma 1 , implies $b=0$. This shows that both $\left\{T x_{n}\right\}$ and $\left\{S x_{n}\right\}$ are Cauchy sequences.

As $T x_{n} \in K$ and $K$ is a closed subset of the Banach space $E$, we conclude that

$$
\lim _{n \rightarrow \infty} T x_{n}=p \in K \text {. }
$$

Since

$$
d\left(T x_{n}, S x_{n}\right) \leq b(n) \rightarrow 0 \quad \text { as } \quad n \rightarrow \infty
$$

we also have $\lim S x_{n}=p$. As $T$ is continuous, we obtain

$$
\lim _{n \rightarrow \infty} T\left(S x_{n}\right)=T\left(\lim _{n \rightarrow \infty} S x_{n}\right)=T p \in K,
$$

and in view of the weak commutativity (3.3), we have

$$
\begin{aligned}
d\left(S T x_{n}, T p\right) & \leq d\left(S T x_{n}, T S x_{n}\right)+d\left(T S x_{n}, T p\right) \leq \\
& \leq d\left(T x_{n}, S x_{n}\right)+d\left(T S x_{n}, T p\right) \rightarrow 0, \text { as } n \rightarrow \infty .
\end{aligned}
$$

This shows that

$$
\lim _{n \rightarrow \infty}(S T)\left(x_{n}\right)=T p
$$

and therefore, by (3.9) and (3.10), we have

$$
M\left(T x_{n}, p\right) \rightarrow d(T p, S p) \quad \text { as } \quad n \rightarrow \infty
$$

and

$$
d(T p, S p) \leq \varphi(d(T p, S p)),
$$

which, again by Lemma 1 , yields $d(T p, S p)=0$, i. e.,

$$
T p=S p .
$$

We shall prove that $S p$ (and also $T p$ ) is a common fixed point for $S$ and $T$. Indeed, by (3.11) and (3.3) it results that

$$
T S p=S T p=S S p .
$$


Now, by (3.1), (3.11), and (3.12), we have

$$
d(S S p, S p) \leq \varphi(M(S p, p))=\varphi(d(S S p, S p)),
$$

which yields $S S p=S p$. It follows from (3.12) that $S p$ is a fixed point of $T$ as well. To prove the uniqueness, relation (3.1) is used.

Remarks. $1^{\circ}$ For $T=1_{E}$ (the identity map) and $\varphi$ given by (1.5), from Theorem 1 we obtain Theorem $\mathrm{C} 2$ of Ćirić.

$\mathbf{2}^{\circ}$ For $\varphi(t)=\lambda t$ with $0<\lambda<1$, Theorem 1 implies Theorem 2 of Rakočević [9]. It is known (see Lemma 4.3.1 in [11]) that if $T$ is a generalized strict $\varphi$-contraction, i. e., $T$ satisfies (1.6) with $E(x, y) \equiv C(x, y)$, and $\varphi$ is a strict comparison function, then $T$ has bounded orbits.

It is, however, an open question whether or not two mappings $S$ and $T$ satisfying (3.1) have bounded orbits.

$\mathbf{3}^{\circ}$ By considering other comparison functions in Theorem 1, we obtain various related fixed point theorems as well as common fixed point theorems. Moreover, all the results in Rakočević [9] can be extended in a similar way. We restrict our study to Theorem 3.

$4^{\circ}$ The continuity assumption of $T$ in Theorem 1 can be weakened to obtain a more general result similar to Theorem 3 of Rakočević [9].

Theorem 2. Let $E$ be a Banach space, $K$ a nonempty closed subset of $E$, and $\partial K \neq \varnothing$ the boundary of $K$. Let $S: K \rightarrow E, T: E \rightarrow E$, and $T: K \rightarrow K$.

Suppose that $T^{m}$ is continuous for some fixed positive integer $m, S$ and $T$ satisfy (3.1), (3.4), (3.5), (3.6), and, moreover, $T$ and $S$ commute, i. e.,

$$
T S x=S T x \text { for every } x \in K .
$$

Then $S$ and $T$ have a unique common fixed point in $K$, provided that $T$ and $S$ have bounded orbits.

Proof. Let $\left\{x_{n}\right\}, S x_{n}$ and $T x_{n}$ be constructed as in the proof of Theorem 1. Hence,

$$
\lim _{n \rightarrow \infty} S x_{n}=\lim _{n \rightarrow \infty} T x_{n}=p \in K .
$$

For every $n \geq 1$, we have

$$
\begin{aligned}
d\left(T^{m} S x_{n}, S T^{m-1} p\right)= & d\left(S T^{m} x_{n}, S T^{m-1} p\right) \leq \\
\leq & \varphi\left(M\left(T^{m} x_{n}, T^{m-1} p\right)\right) \\
= & \varphi\left(\operatorname { m a x } \left\{d\left(T^{m} T x_{n}, T^{m} p\right), d\left(T^{m} T x_{n}, T^{m} S x_{n}\right),\right.\right. \\
& \left.\left.d\left(T^{m} p, S T^{m-1} p\right), d\left(T^{m} T x_{n}, S T^{m-1} p\right), d\left(T^{m} p, T^{m} S x_{n}\right)\right\}\right) .
\end{aligned}
$$

Then, by the continuity of $T^{m}$,

$$
d\left(T^{m} p, S T^{m-1} p\right) \leq \varphi\left(d\left(T^{m} p, S T^{m-1} p\right)\right),
$$


and hence $T^{m} p=S T^{m-1} p$, by Lemma 1 . So, similarly to the method used in the proof of Theorem 1, it can be shown that $T^{m} p$ is a common fixed point of $T$ and $S$. The uniqueness is proved by using the contraction condition (3.1).

$\mathrm{p}$

\section{Acknowledgements}

The author would like to thank the referee for his helpful comments and suggestions towards improving the paper as well as for pointing out the need of the assumption on bounded orbits in Theorems 1 and 2.

\section{REFERENCES}

[1] Berinde, V.: Generalized Contractions and Applications, Editura Cub Press 22, Baia Mare, 1997 (in Romanian).

[2] Berinde, V.: Iterative Approximation of Fixed Points, Editura Efemeride, Baia Mare, 2002.

[3] Browder, F. E.: On the convergence of successive approximations for nonlinear functional equations, Indagat. Math., 30 (1968), 27-35.

[4] Ćirić, LJ. B.: A generalization of Banach's contraction principle, Proc. Amer. Math. Soc., 45 (1974), 267-273.

[5] ĆIRIć, Lu. B.: Quasi contraction nonself mappings on Banach spaces, Bull. Acad. Serbe Sci. Arts, Cl. Sci. Math. Natur. Sci. Math., 23 (1998), 25-31.

[6] Kannan, R.: Some results on fixed points, Bull. Calcutta Math. Soc., 60 (1968), 71-76.

[7] MÉszáros, J.: A comparison of various definitions of contractive type mappings, Bull. Calcutta Math. Soc., 84 (1992), No. 2, 167-194.

[8] Radovanović, V.: Approximation of a fixed point of some nonself mappings, Math. Balkanica, 15 (2001), 213-218.

[9] RAKočEvić, V.: Quasi contraction nonself mappings on Banach spaces and common fixed point theorems, Publ. Math. Debrecen, 58 (2001), No. 3, 451-460.

[10] Rhoades, B. E.: Contractive definitions and continuity, Contemporary Math., 72 (1988), 233-245.

[11] Rus, I. A.: Generalized Contractions and Applications, Cluj University Press, Cluj-Napoca, 2001.

[12] Walter, W.: Remarks on a paper by F. Browder about contraction, Nonlinear Anal. TMA, 5 (1981), 21-25.

\section{Author's Address}

Vasile Berinde:

Department of Mathematics and Computer Science, North University of Baia Mare, Victoriei 76, 430072 Baia Mare, Romania

E-mail address: vberinde@ubm.ro; vasile_berinde@yahoo.com 